\title{
Avaliação do Parceiro Sexual e Risco de Recidivas em Mulheres Tratadas por Lesões Genitais Induzidas por Papilomavirus Humano (HPV)
}

\author{
Male Sexual Partner Evaluation and Relapse Risk in Women Treated for \\ Genital Human Papillomavirus (HPV) Lesions
}

Júlio César Teixeira, Sophie Françoise Mauricette Derchain, Luiz Carlos Teixeira César Cabello dos Santos, Kazue Panetta, Luiz Carlos Zeferino

\section{RESUMO}

\begin{abstract}
Objetivo: estudar a associação entre a avaliação ou não do parceiro e recidivas em mulheres tratadas por lesões por HPV.

Métodos: estudo de coorte reconstituido com 144 mulheres com lesões por HPV e cujos parceiros foram avaliados, comparadas com 288 mulheres cujos parceiros não foram examinados, controladas por data do atendimento, idade, grau das lesões e tratamento, todos atendidos entre julho/1993 e março/2000. Avaliaram-se a semelhança entre os grupos, a associação entre a avaliação ou não dos parceiros ou o diagnóstico de lesões com a ocorrência e grau das recidivas nas mulheres e o tempo livre de doença (TLD).

Resultados: os grupos foram semelhantes com relação às variáveis de controle. Encontrouse 9,0\% de recidivas nas mulheres cujos parceiros foram avaliados e 5,9\% quando os parceiros não foram ( $p=0,23)$. Quando se diagnosticaram lesões nos homens, $12,5 \%$ das suas parceiras apresentaram recidivas contra 7,3\% das parceiras de homens sem lesões ( $p=0,23)$, mas sem correlação com o grau da lesão recidivada e TLD. Quando o homem referiu relação conjugal monogâmica de até 12 meses, observou-se 14,9\% de recidivas nas mulheres, contra $6,2 \%$ para as mulheres cujo parceiro relatou tempo maior $(p=0,08)$.

Conclusões: a avaliação do homem não diminuiu o risco de recidivas de lesões por HPV na parceira. A presença de lesões nos parceiros não se correlacionou com a ocorrência e grau das recidivas nas mulheres e TLD. Estas observações não suportam a hipótese de que os homens não avaliados seriam importante causa de recidivas nas parceiras.
\end{abstract}

PALAVRAS-CHAVE: HPV. Infecção genital. DST. Peniscopia.

\section{Introdução}

A infecção genital por papilomavírus humano (HPV) é uma das doenças sexualmente transmissiveis (DST) mais freqüentes, acometendo cerca de $30 \%$ da população sexualmente ativa ${ }^{1-5}$. Num contexto de alta prevalência, infectividade e diagnósticos na forma de lesões subclínicas e assintomáticas, fica evidente que a disseminação do HPV

Área de Oncologia Ginecológica e Patologia Mamária do Departamento de Tocoginecologia da Faculdade de Ciências Médicas e Centro de Atenção Integral à Saúde da Mulher (CAISM) da Universidade Estadual de Campinas (UNICAMP). Correspondência:

Júlio César Teixeira

Rua Barão Geraldo de Rezende, 282 conj. 61

13.020-440 - Campinas - SP

e-mail: julioc@directnet.com.br tende a ser universal entre os sexualmente ativos, sendo o homem um importante meio propagador deste vírus entre as mulheres ${ }^{6-9}$. É comum que os médicos que atendem mulheres com lesões genitais por HPV, devido ao seu potencial oncogênico, por não ter tratamento específico e por tratar-se de DST, sintam a necessidade de avaliar o parceiro sexual para pesquisar a presença de lesões semelhantes e diminuir o risco de recidivas $^{5,7,8,10-13}$. Esta avaliação é realizada por meio da peniscopia, que começou a ser desenvolvida por Levine et al. ${ }^{10}$. É exame pouco específico e o diagnóstico final é confirmado pela avaliação histopatológica em tecidos de biópsia em cerca de 30 a $65 \%$ dos parceiros ${ }^{1,7,13-15}$. Por outro lado, outros autores afirmam que, após a mulher já ter tido o contágio, o surgimento de uma lesão e sua 
evolução estariam diretamente ligados ao confronto entre o vírus e o hospedeiro, tendo o sistema imunológico um importante papel ${ }^{16,17}$. As recidivas nas mulheres tratadas de lesões genitais por HPV ocorrem, mas a avaliação dos seus parceiros sexuais não é solicitada de forma sistemática e, quando avaliados, somente cerca de um terço apresentam lesões e com maior probabilidade de evolução benigna. Assim, fica dúvida sobre a real necessidade de examinar o parceiro e sua influência na recidiva de lesões nas mulheres ou na prevenção de câncer genital. O propósito deste estudo é avaliar qual o impacto do exame dos parceiros sexuais no seguimento de mulheres tratadas por lesões genitais induzidas por HPV.

\section{Pacientes e Métodos}

Realizamos estudo de coorte reconstituído com um grupo formado por 144 mulheres tratadas por lesões genitais induzidas por HPV, com seguimento mínimo de um ano e cujos parceiros foram avaliados por meio da peniscopia, conforme estudo previamente publicado por Teixeira et al. $(1999)^{18}$, tratados, se necessário, e seguidos por no minimo seis meses. O grupo controle totalizando 288 mulheres foi formado selecionando-se duas mulheres para cada uma do grupo inicial, controlando-se a data do atendimento, a idade e o grau mais grave da lesão intra-epitelial genital, independentemente do local, se colo uterino, vagina ou vulva. Deviam ter também tempo de seguimento mínimo de um ano e ausência de anotações nos prontuários de que seus parceiros tenham sido examinados em qualquer momento. Indiretamente, esperou-se controlar o tipo de tratamento (cauterizações elétrica ou química, conização do colo uterino (a frio ou por cirurgia de alta freqüência (CAF) com alça), histerectomia total ou cirurgias na vagina ou vulva), o hábito de fumar e algumas características epidemiológicas, como a idade no início da atividade sexual e o número de parceiros. Assim, definimos que a avaliação ou não dos parceiros tenha sido a única diferença importante entre os grupos. Para esta análise inicial, foram utilizados os testes exato de Fisher e $t$ de Student para verificarmos a semelhança entre os grupos (Tabela 1). Todos os pacientes foram atendidos no Ambulatório do Programa de Controle do Carcinoma Cérvico-Uterino da Área de Oncologia Ginecológica do Departamento de Tocoginecologia da Faculdade de Ciências Médicas e Centro de Atenção Integral à Saúde da Mulher (CAISM) da Universidade Estadual de Campinas (UNICAMP), entre julho de 1993 e julho de 1999 e seguidos até março de 2000.

Tabela 1 - Comparação entre os grupos estudados de acordo com as variáveis de controle.

\begin{tabular}{|c|c|c|c|}
\hline \multirow{3}{*}{ Variáveis } & \multicolumn{2}{|c|}{ Mulheres com parceiros } & \multirow[b]{3}{*}{$\mathrm{p}$} \\
\hline & Avaliados & Não avaliados & \\
\hline & $n=144$ & $n=288$ & \\
\hline Média e (DP) & $27,1(8,2)$ & $27,4(8,2)$ & $0,72^{(1)}$ \\
\hline Variação & $14-63$ & $14-61$ & \\
\hline Número de parceiros sexuais: média e (DP) & $2,6(3,1)^{(3)}$ & $3,0(2,8)^{(2)}$ & $0,21^{(1)}$ \\
\hline Tabagismo (\%) & $(34,3)$ & $(40,2)$ & $0,10^{(4)}$ \\
\hline \multicolumn{4}{|l|}{ Grau da lesão (\%) } \\
\hline 1 (NIC, NIVA ou NIV 1) & $(60,4)$ & $(58,0)$ & \\
\hline 2 (NIC, NIVA ou NIV 2) & $(13,9)$ & $(11,1)$ & \\
\hline Cauterizações & $(61,8)$ & $(59,0)$ & \\
\hline Conização de colo uterino & $(31,9)$ & $(36,1)$ & \\
\hline Histerectomia & $(4,2)$ & $(4,2)$ & \\
\hline Cirurgias na vagina ou vulva & $(2,1)$ & $(0,7)$ & $0,54^{(4)}$ \\
\hline
\end{tabular}


As mulheres foram consideradas curadas quando apresentaram controle negativo pelos exames ginecológico, colposcópico e citológico em até três meses após o término do tratamento. Nova lesão diagnosticada pela histopatologia após este período e em qualquer localização do trato genital inferior foi considerada recidiva. Correlacionamos, então, a ocorrência destas recidivas nas mulheres de acordo com a realização ou não da avaliação do parceiro. Depois, avaliamos a proporção de pacientes livres de recidivas aos seis meses, um ano e ao final de dois anos com relação aos grupos estudados, por meio de análise de sobrevivência de Kaplan e Meier e do teste de Wilcoxon. Posteriormente, para as mulheres cujos parceiros foram examinados, avaliamos a associação entre a ocorrência de recidivas, o grau da lesão na recidiva e o tempo livre de doença com a presença de lesões por HPV nos homens e com o tempo de estabilida-

Tabela 2 - Distribuição percentual da ocorrência de recidivas de lesões induzidas por HPV nas mulheres, segundo a avaliação ou não do parceiro sexual.

\begin{tabular}{lrrrr}
\hline Recidiva & \multicolumn{4}{c}{ Mulheres com parceiros } \\
& \multicolumn{2}{c}{ Avaliados } & Não avaliados \\
& $\mathbf{n}$ & $(\%)$ & $\mathbf{n}$ & $(\%)$ \\
\hline Sim & 13 & $(9,0)$ & 17 & $(5,9)$ \\
Não & 131 & $(91,0)$ & 271 & $(94,1)$ \\
Total & 144 & $(100,0)$ & 288 & $(100,0)$ \\
\hline$p=0,23$ (teste exato de Fisher). & & &
\end{tabular}

Tabela 3 - Estimativas da proporção de mulheres livres de recidivas, segundo a avaliação ou não do parceiro sexual.

\begin{tabular}{lccccc}
\hline Mulheres com parceiros & $\mathbf{n}$ & Recidiva & \multicolumn{3}{c}{ Proporção de mulheres livres de recidiva (\%) } \\
& & & Após 6 meses & Após 1 ano & Após 2 anos \\
\hline Avaliados & 144 & 13 & 99,3 & 96,5 & 87,9 \\
Não avaliados & 288 & 17 & 100,0 & 97,2 & 92,7 \\
Total & 432 & 30 & 99,8 & 97,0 & 91,2 \\
\hline
\end{tabular}

$p=0,17$ (teste de Wilcoxon).

Ao analisarmos somente as mulheres cujos parceiros foram avaliados, observamos que ocorreram $12,5 \%$ de recidivas nestas quando seus parceiros apresentaram lesões por HPV contra 7,3\% de recidivas nas mulheres com avaliação do parceiro negativa, diferença esta estatisticamente de conjugal masculina. Para a análise estatística, adotou-se o limite de 5\% para significância. Esta pesquisa foi aprovada pelo Comitê de Ética em Pesquisa do CAISM, UNICAMP.

\section{Resultados}

A ocorrência de recidivas de lesões induzidas por HPV foi um pouco mais freqüente nas mulheres cujos parceiros foram avaliados $(9,0 \%)$ no que naquelas cujos parceiros não foram avaliados (5,9\%), não sendo esta diferença significante $(p=0,23$, Tabela 2). Ao avaliarmos as estimativas de tempo para que as recidivas tivessem ocorrido, não foram observadas diferenças significativas entre os grupos estudados (Tabela 3 e Figura 1).

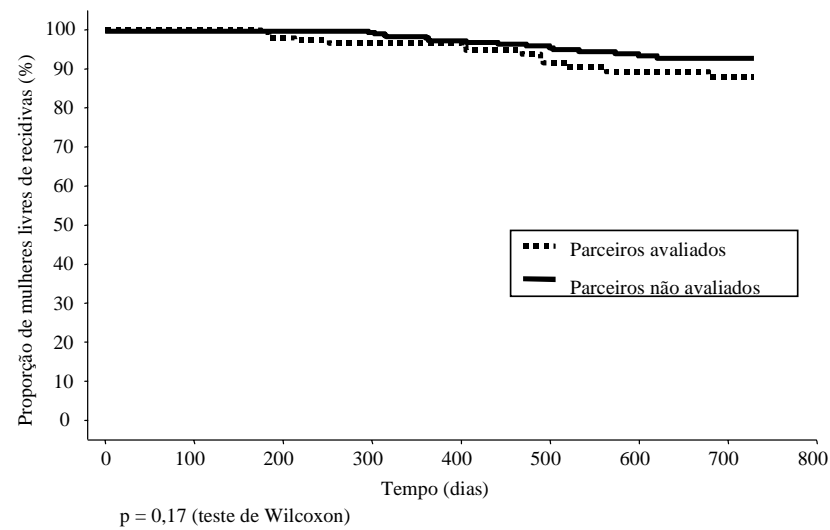

Figura 1 - Estimativa da proporção de mulheres livres de recidivas, segundo a avaliação ou não do parceiro sexual. 
Tabela 4 - Distribuição percentual das recidivas, do grau destas lesões e do tempo livre de doença nas mulheres cujos parceiros foram avaliados, segundo a presença ou não de lesões por HPV nos homens.

\begin{tabular}{|c|c|c|c|c|c|}
\hline \multirow[t]{3}{*}{ Variáveis } & \multicolumn{4}{|c|}{ Lesão por HPV no homem } & \multirow[b]{3}{*}{$\mathbf{p}^{(1)}$} \\
\hline & \multicolumn{2}{|c|}{ Sim } & \multicolumn{2}{|c|}{ Não } & \\
\hline & $\mathrm{n}$ & $(\%)$ & $\mathrm{n}$ & (\%) & \\
\hline \multicolumn{6}{|c|}{ Recidiva na mulher $(n=144)$} \\
\hline Sim & 6 & $(12,5)$ & 7 & $(7,3)$ & 0,23 \\
\hline Não & 42 & $(87,5)$ & 89 & $(92,7)$ & \\
\hline Total & 48 & $(100,0)$ & 96 & $(100,0)$ & \\
\hline \multicolumn{6}{|c|}{ Grau da lesão na recidiva $(n=13)$} \\
\hline 1 & 5 & (83) & 4 & (57) & 0,34 \\
\hline 2 ou 3 & 1 & $(17)$ & 3 & (43) & \\
\hline Total & 6 & $(100)$ & 7 & $(100)$ & \\
\hline \multicolumn{6}{|c|}{ Tempo livre de doença $(n=13)$} \\
\hline Até 12 meses & 2 & (33) & 3 & (43) & 0,59 \\
\hline$>12$ meses & 4 & $(67)$ & 4 & (57) & \\
\hline Total & 6 & $(100)$ & 7 & $(100)$ & \\
\hline
\end{tabular}

(1) teste exato de Fisher.

\section{Discussão}

A ocorrência de recidivas durante o seguimento das mulheres tratadas por lesões genitais induzidas por HPV e que tiveram seus parceiros avaliados e tratados foi de $9,0 \%$, contra $5,9 \%$ para aquelas mulheres cujos parceiros não foram examinados, e esta diferença não esteve associada à realização ou não desta avaliação. Observou-se uma tendência de que um tempo de estabilidade conjugal masculina menor ou igual a 12 meses estaria associado com duas vezes mais recidivas nas mulheres que aquelas cujos parceiros relataram um tempo de estabilidade conjugal maior. A ocorrência, o grau da lesão recidivada e o tempo livre de doença não estiveram associados à presença ou não de lesões induzidas por HPV nos homens.

Procuramos definir dois grupos de mulheres os mais semelhantes possiveis com relação às variáveis associadas com o surgimento e com as recidivas de lesões genitais por HPV, para podermos inferir uma possivel associação entre a ocorrência de recidivas na mulher com a avaliação ou não dos seus parceiros sexuais.

Alguns autores sugerem que as chances de cura e o risco de recidivas destas lesões nas mulheres poderiam estar associados à presença ou não de lesões nos seus parceiros sexuais ${ }^{7,8,10-13}$. Porém, neste estudo, a ocorrência de recidivas durante o seguimento não diminuiu com a realização desta avaliação e, ao contrário, houve um pouco mais de recidivas naquelas mulheres cujos parceiros foram avaliados e tratados.

Achados semelhantes foram relatados por Krebs e Helmkamp ${ }^{19}$ que compararam 190 casais com 200 mulheres controladas por idade, grau da displasia cervical e tipo de tratamento realizado, mas cujos parceiros não tinham sido avaliados. Os autores observaram que a incidência de recidivas foi de $6,8 \%$ para as mulheres cujos parceiros foram examinados e tratados contra $7,5 \%$ para o grupo controle. Posteriormente, em outro estudo semelhante realizado com portadores de condiloma acuminado, os mesmos autores compararam outros 180 casais com 180 mulheres, encontrando incidência de recidivas de $16,7 \%$ para as mulheres cujos parceiros foram examinados e tratados contra $18,9 \%$ para o grupo controle ${ }^{20}$. Baseados nestes estudos, os autores sugerem que uma reinfecção mediante o mesmo parceiro sexual não seria importante causa de recidiva de lesões por HPV em mulheres.

Recentemente, Syrjanen e Syrjanen ${ }^{5}$ relataram resultados preliminares de um estudo para avaliar a influência do tratamento dos homens na evolução clínica da infecção por HPV nas parceiras, no qual 533 casais foram divididos em quatro grupos: 256 casais com homens não avaliados; 105 casais com homens sem HPV; 74 casais com homens com HPV e tratados; 98 casais com homens com HPV e não tratados. Após dois anos de seguimento, verificaram que as recidivas nas mulheres ocorreram entre $1,0 \%$ e 5,9\% nos diversos grupos e não estiveram associadas à avaliação ou tratamento dos seus parceiros. Com estes resultados, os autores mostraram que os altos índices de cura alcançados nas mulheres não foram melhorados por exames e tratamentos dos parceiros.

Em face da nova lesão, não é possível definir se se trata de nova infecção ou reativação de infecção latente ou recidiva ${ }^{21}$. Algumas recidivas apresentam, claramente, associação com exposi- 
ção recente por meio de relações sexuais com parceiros diferentes, confirmando o que sugerem alguns estudos, nos quais a ocorrência de uma infecção viral estaria fortemente associada ao número de parceiros sexuais, principalmente no último ano avaliado ${ }^{4,9,17}$. Indiretamente, tempo de estabilidade conjugal menor também poderia estar associado, como observado neste estudo.

Observa-se que nem todos os parceiros são encaminhados para avaliação. Esta possibilidade geralmente é lembrada diante de caso mais grave ou, principalmente, quando há freqüentes recidivas na mulher ou dificuldades terapêuticas. Sendo assim, é provável que esta apresente por si só e independentemente da avaliação e tratamento de seus parceiros, maior risco de evolução desfavorável e com recidivas. Neste estudo, as mulheres cujos parceiros foram avaliados poderiam ser de pior prognóstico, aumentando o risco de recidivas, o que poderia estar compensando uma possível proteção obtida com esta avaliação.

Somente cerca de um terço dos parceiros apresentam lesões genitais, sendo geralmente subclínicas e, apesar do potencial de disseminação do HPV, apresentando baixo risco de evolução, sendo a cura espontânea o principal desfecho nos homens ${ }^{14,18,22}$.

A peniscopia é exame pouco específico e requer aprendizado contínuo e acúmulo de experiências em como realizá-la de forma mais prática, valorizando os achados com base na epidemiologia e no caso em particular. O diagnóstico só deveria ser firmado após confirmação histopatológica em tecidos de biópsias, para evitar diagnósticos exagerados e tratamentos desnecessários ${ }^{5,14,18}$.

Apesar de a avaliação do parceiro não ter apresentado associação com uma redução no número de recidivas, o diagnóstico de lesões por HPV nos homens poderia se associar à ocorrência de recidivas, com o tempo livre de doença ou com o grau das lesões recidivadas. Neste estudo, de forma semelhante aos resultados de Krebs e Helmkamp ${ }^{19},{ }^{20}$ e Hippelainen et al. ${ }^{22}$, não foram observadas associações neste sentido, sugerindo que estes fatos não estariam relacionados com novo aporte de vírus para as mulheres por meio da relação sexual com seus parceiros.

Entre as mulheres, após uma infecção, a evolução seria determinada somente pelo equilíbrio entre fatores virais e do hospedeiro ${ }^{16,17}$. Nova carga viral do mesmo tipo de HPV adquirida por relação sexual de parceiro com lesão genital parece não influenciar nas recidivas ou progressão das lesões, a não ser pela possibilidade de tratarse de um contato sexual com um tipo de HPV diferente daqueles já adquiridos pela paciente e aí possibilitar o surgimento, não de recidivas, mas de novas lesões ${ }^{20,23}$. Desta forma, deve-se salientar aos casais que a estabilidade conjugal seria mais importante na prevenção e controle desta patologia que avaliações e tratamentos sistemáticos dos parceiros.

Como a avaliação do homem neste estudo não trouxe, aparentemente, benefícios à mulher, poder-se-ia indicar a peniscopia para beneficiá-lo ou diminuir a disseminação viral na população. Assim, juntamente com o baixo potencial oncogênico de infecção genital por HPV nos homens, esta avaliação poderia ser realizada de forma simplificada, focalizando-se a detecção e tratamento de lesões clínicas. O tempo restante seria investido em orientações, ressaltando a importância de se manter estabilidade conjugal, pois o uso de preservativos, apesar de ser eficaz contra outras DST, não protege eficientemente contra o HPV.

Após a eliminação das lesões clínicas ou suspeitas nos homens ou naqueles com avaliação negativa, não haveria necessidade de seguimento rigoroso ou por tempo prolongado, talvez com exceção dos casos de carcinoma in situ do pênis.

Desta forma, o momento ideal de encaminhamento destes parceiros de risco parece ser por ocasião do diagnóstico inicial na mulher, confirmado na histopatologia. Neste momento, além de se realizar adequada orientação do casal, deve-se esclarecer a mulher de que a lesão por HPV pode ser decorrente de infecção acontecida há anos e que o seu curso, na maioria dos casos, é benigno, afastando-se o temor de câncer. Com isso, procura-se criar quadro psicológico positivo, gerando tranqüilidade e confiança, resultando em auxílio na cura e mantendo a harmonia conjugal.

Concluindo, enquanto aguardamos novas terapêuticas e as vacinas, algumas em fase três de experimentação, devemos insistir na avaliação do parceiro sexual de forma simplificada, com alta resolutividade, evitando-se retornos e supertratamentos, na época do diagnóstico inicial da mulher confirmado pela histopatologia, não havendo necessidade do reencaminhamento dos homens se ocorrerem recidivas nas parceiras de casais com união estável e, principalmente, utilizar esta oportunidade para adequada orientação individual e do casal.

\section{ABSTRACT}

Purpose: to study the association between the evaluation or not of the male partner and relapses in women treated for HPV lesions.

Methods: a reconstituted cohort study with 144 women with HPV lesions and whose partners had been evaluated, compared with 288 women whose partners had not been examined, controlled regarding date of attendance, age, lesion degree and treatment, attended between July 1993 and March 2000. We evaluated similarity between the groups, the association between the evaluation or not of the partners or the diagnosis of lesions with the occurrence and relapse lesion degree in the 
women and the disease-free interval (DFI).

Results: the groups were similar regarding the control variables. Relapses occurred in $9.0 \%$ of the women whose partners had been evaluated and in $5.9 \%$ of those whose partners had not been evaluated ( $p=0.23)$. When lesions were diagnosed in the men, $12.5 \%$ of their partners had relapses, against $7.3 \%$ of the women whose partners had no lesions $(p=0.23)$, but there was no correlation with the relapse lesion degree and DFI. When the men reported a time of monogamous conjugal relationship $\leq 12$ months, we observed $14.9 \%$ relapses in women, against $6.2 \%$ for the women whose partners reported a longer time $(p=0.08)$.

Conclusions: evaluation of the man did not decrease the relapse risk of HPV lesions in his partner. The presence of lesions in the male partners did not correlate with the occurrence and relapse degree in women and DFI. This study does not support the hypothesis that nonevaluated men would be an important cause of relapses in their partners.

KEY WORDS: HPV. Genital infection. STD. Peniscopy.

\section{Referências}

1. Hippelainen M, Syrjanen S, Hippelainen M, et al. Prevalence and risk factors of genital human papillomavirus (HPV) infections in healthy males: a study on Finnish conscripts. Sex Transm Dis 1993; 20:321-8.

2. Derchain S, Neves-Jorge JP, Andrade L, Pinto-Neto AM, Silva JP. Infection by human papillomavirus in sexually active teenagers: clinic and subclinic manifestations. São Paulo Med J 1995; 113:948-52.

3. Franco EL, Villa LL, Ruiz A, Costa MC. Transmission of cervical human papillomavirus infection by sexual activity: differences between low and high oncogenic risk types. J Infect Dis 1995; 172:756-63.

4. Karlsson R, Jonsson M, Edlund K, et al. Lifetime number of partners as the only independent risk factor for human papillomavirus infection: a populationbased study. Sex Transm Dis 1995; 22:119-27.

5. Syrjanen KJ, Syrjanen SM. Papillomavirus infections in human pathology. 1st ed. Chichester: John Wiley \& Sons; 1999.

6. Zunzunegui MV, King MC, Coria CF, Charlet J. Male influences on cervical cancer risk. Am J Epidemiol 1986; 123:302-7.

7. Boon ME, Schneider A, Hogewoning CJ, van der Kwast TH, Bolhuis P, Kok LP. Penile studies and heterosexual partners: peniscopy, cytology, histology, and immunocytochemistry. Cancer 1988; 61:1652-9.

8. Syrjanen K, Syrjanen S. Epidemiology of human papilloma virus infections and genital neoplasia. Scand J Infect Dis Suppl 1990; 69:7-17.

9. Burk RD, Ho GY, Beardsley L, Lempa M, Peters M, Bierman R. Sexual behavior and partner characteristics are the predominant risk factors for genital human papillomavirus infection in young women. J Infect Dis 1996; 174:679-89.
10.Levine RU, Crum CP, Herman E, Silvers D, Ferenczy A, Richart RM. Cervical papillomavirus infection and intraepithelial neoplasia: a study of male sexual partners. Obstet Gynecol 1984; 64:16-20.

11.Barrasso R, De Brux J, Groissant O, Orth G. High prevalence of papillomavirus-associated penile intraepithelial neoplasia in sexual partners of women with cervical intraepithelial neoplasia. $\mathrm{N}$ Engl J Med 1987; 317:916-23.

12.Bergman A, Nalick R. Prevalence of human papillomavirus infection in men: comparison of the partners of infected and uninfected women. J Reprod Med 1992; 37:710-2.

13.Frega A, Stentella P, Villani C, et al. Correlation between cervical intraepithelial neoplasia and human papillomavirus male infections: a longitudinal study. Eur J Gynaecol Oncol 1999; 20:228-30.

14. Koronel R, Stefanon B, Pilotti S, Bandieramonte G, Rilke F, De Palo G. Genital human papilloma virus infection in males. A clinico-pathologic study. Tumori 1991; 77:76-82.

15. Nicolau SM, Martins NV, Ferraz PE, et al. Importance of peniscopy, oncologic cytology and histopathology in the diagnosis of penile infection by human papillomavirus. São Paulo Med J 1997; 115:1330-5.

16. Coleman N, Birley HD, Renton AM, et al. Immunological events in regressing genital warts. Am J Clin Pathol 1994; 102:768-74.

17.Ho GY, Bierman R, Beardsley L, Chang CJ, Burk RD. Natural history of cervicovaginal papillomavirus infection in young women. N Engl J Med 1998; 338:423-8.

18. Teixeira JC, Santos CC, Derchain SFM, Zeferino LC. Lesões induzidas por papilomavírus humano em parceiros de mulheres com neoplasia intra-epitelial do trato genital inferior. Rev Bras Ginecol Obstet 1999; 21:431-7.

19.Krebs HB, Helmkamp BF. Does the treatment of genital condylomata in men decrease the treatment failure rate of cervical dysplasia in the female sexual partner? Obstet Gynecol 1990; 76:660-3.

20.Krebs HB, Helmkamp BF. Treatment failure of genital condylomata acuminata in women: role of the male sexual partner. Am J Obstet Gynecol $1991 ; 165: 337-40$.

21.Franco EL, Villa LL, Sobrinho JP, et al. Epidemiology of acquisition and clearance of cervical human papillomavirus infection in women from a high-risk area for cervical cancer. J Infect Dis 1999; 180:141523.

22.Hippelainen MI, Hippelainen M, Saarikoski S, Syrjanen K. Clinical course and prognostic factors of human papillomavirus infections in men. Sex Transm Dis 1994; 21:272-9.

23.Hippelainen MI, Yliskoski M, Syrjanen S, et al. Low concordance of genital human papillomavirus (HPV) lesions and viral types in HPV-infected women and their male sexual partners. Sex Transm Dis 1994; 21:76-82. 